\title{
Correction: Spotlight on South America
}

Nature Ecology \& Evolution 1, 0129 (2017); published 23 March 2017; corrected 28 April 2017.

The original image that was published with this Editorial was not of the intended species. This has been replaced in all versions of the Editorial with a photo of Oophaga lehmanni by Andres Posso-Terranova. 